\title{
Two-dimensional numerical models for overland flow simulations
}

\author{
P. Costabile, C. Costanzo \& F. Macchione \\ LAMPIT, Dipartimento di Difesa del Suolo, University of Calabria, Italy
}

\begin{abstract}
In this paper the performances of a number of two-dimensional (2D) models for overland flow simulations have been compared; in particular they are based on the shallow water approach according to a fully dynamic, diffusive and kinematic wave modelling. The systems have been numerically solved by using both the MacCormack second order central scheme and HLL first order upwind scheme. The numerical results highlighted that the differences among the simulations are not very important when the simulations refer to commonly used ideal literature tests in which the topography is quite simplified while significant differences have been observed when the topography is more similar to the real situations.
\end{abstract}

Keywords: flood modelling, overland flow, 2D models.

\section{Introduction}

The analysis of flood events due to heavy rainfall needs a strict and innovative methodology capable to provide the evolution of the phenomena in relation with potential extreme events as well as their spatial and temporal distribution within a selected area.

In order to obtain a reliable prediction of the hydraulic risk associated to extreme events, the use of numerical simulation models, appropriately validated using both experimental and real event data, seems to be necessary. Such models are able to carry out a quantitative evaluation of the most important parameters in the context of the hydraulic protection of the territory as water depths, velocities and flooded areas.

At the present time, there is a tendency to develop more and more accurate models to manage the risk associated with potential extreme meteorological 
events at basin scale especially in the case of flat valleys where even a slight error in modelled water surface elevations may lead to large errors in the prediction of inundated areas (Sanders [17]). This is true also in the context of the prediction of the climate changes consequences: in order to obtain a reliable tool of analysis it is necessary to couple a meteorological model with an hydrodynamic model, both at high resolution, defining the components of a hydro-meteorological chain. In this context, the 2D fully dynamic shallow water equations seem to be the required approach to deal with that situation because it allows one to analyse in depth the flow behaviour in locally complex topography. On the other hand, the use of different approximations of the unsteady flow equations is very common in order to simulate the overland flow processes leading to the kinematic and diffusive wave models $[1,3,7,9,12]$. Several authors have studied the conditions in which those approximations are completely justified [14-16, 21]. A comprehensive review of the applicability criteria may be found in Tsai [20] in which the backwater effects have been also included in the analysis.

The previous studies have been always carried out using a very simplified idealised topography, simulating the complex hillslopes as plane surfaces with constant hydraulic properties. The numerical models themselves are quite often validated with reference to theoretical solutions in which the topography is dramatically simplified.

One of the purposes of this paper is to analyse in depth the performances of overland flow models. In particular, models based on fully dynamic, diffusive and kinematic wave approach have been developed, tested and validated with the numerical tests commonly used in the literature. The numerical integration has been carried out using both a first order upwind (HLL scheme) and a II order central scheme (MacCormack scheme). The performances of the models have been compared and discussed using several numerical tests.

\section{Mathematical model}

The implemented codes are based on the fully conservative shallow water equations:

$$
\frac{\partial \mathbf{U}}{\partial t}+\frac{\partial \mathbf{F}}{\partial x}+\frac{\partial \mathbf{G}}{\partial y}=\mathbf{S}
$$

where:

$$
\mathbf{U}=\left(\begin{array}{l}
h \\
h u \\
h u
\end{array}\right) ; \mathbf{F}=\left(\begin{array}{l}
h u \\
h u^{2}+g h^{2} / 2 \\
h u v
\end{array}\right) ; \mathbf{G}=\left(\begin{array}{l}
h v \\
h u v \\
h v^{2}+g h^{2} / 2
\end{array}\right) ; \mathbf{S}=\left(\begin{array}{l}
r-f \\
g h\left(S_{0 x}-S_{f x}\right) \\
g h\left(S_{0 y}-S_{f y}\right)
\end{array}\right)
$$

in which: $t$ is time; $x, y$ are the horizontal coordinates; $h$ is the water depth; $u, v$ are the depth-averaged flow velocity in $x$ - and $y$-directions; $g$ is the gravitational acceleration; $S_{0 x}, S_{0 y}$ are the bed slopes in $x$ - and $y$-directions; $S_{f x}, S_{f y}$ are the friction slopes in $x$ - and $y$-directions, which can be calculated from Strickler's formula; $r$ is the rain intensity and $f$ are the infiltration losses. 
By neglecting the local and convective acceleration in the momentum conservation equations, it is possible to obtain the following diffusive model:

$$
\frac{\partial \mathbf{U}_{\mathbf{d}}}{\partial t}+\frac{\partial \mathbf{F}_{\mathbf{d}}}{\partial x}+\frac{\partial \mathbf{G}_{\mathbf{d}}}{\partial y}=\mathbf{S}_{\mathbf{d}}
$$

with:

$$
\mathbf{U}_{d}=\left(\begin{array}{l}
h \\
0 \\
0
\end{array}\right) ; \mathbf{F}_{d}=\left(\begin{array}{l}
h u \\
g h^{2} / 2 \\
0
\end{array}\right) ; \mathbf{G}_{d}=\left(\begin{array}{l}
h v \\
0 \\
g h^{2} / 2
\end{array}\right) ; \mathbf{S}_{d}=\left(\begin{array}{l}
r-f \\
g h\left(S_{0 x}-S_{f x}\right) \\
g h\left(S_{0 y}-S_{f y}\right)
\end{array}\right)
$$

and ignoring also the depth gradient terms one may obtain the following kinematic model:

$$
\frac{\partial \mathbf{U}_{\mathbf{k}}}{\partial t}+\frac{\partial \mathbf{F}_{\mathbf{k}}}{\partial x}+\frac{\partial \mathbf{G}_{\mathbf{k}}}{\partial y}=\mathbf{S}_{\mathbf{k}}
$$

with:

$$
\mathbf{U}_{k}=\left(\begin{array}{l}
h \\
0 \\
0
\end{array}\right) ; \mathbf{F}_{k}=\left(\begin{array}{l}
h u \\
0 \\
0
\end{array}\right) ; \mathbf{G}_{k}=\left(\begin{array}{l}
h v \\
0 \\
0
\end{array}\right) ; \mathbf{S}_{k}=\left(\begin{array}{l}
r-f \\
g h\left(S_{0 x}-S_{f x}\right) \\
g h\left(S_{0 y}-S_{f y}\right)
\end{array}\right)
$$

\section{Numerical models}

The finite volume method, widely adopted in the literature, has been used to discretize the previous equations. It considers the integral form of the shallow water equations that allows a quite easy implementation of shock capturing schemes on different mesh type. The system of equations is integrated over an arbitrary control volume $\Omega_{i, j}$ and, in order to obtain surface integrals, the Green theorem has been applied to each component of the flux vectors (for example $\mathbf{F}$ and $\mathbf{G )}$ leading to:

$$
\frac{\partial}{\partial t} \int_{\Omega_{i, j}} \mathbf{U} d \Omega+\oint_{\partial \Omega_{i, j}}[\mathbf{F}, \mathbf{G}] \cdot \mathbf{n} d L=\int_{\Omega_{i, j}} \mathbf{S} d \Omega
$$

where $\partial \Omega_{i, j}$ being the boundary enclosing $\Omega_{i, j}, \mathbf{n}$ is the unit vector normal and $d L$ is the length of each boundary. Denoting by $\mathbf{U}_{i, j}$ the average value of the flow variables over the control volume $\Omega_{i, j}$ at a given time, the equation (16) may be discretized as:

$$
\mathbf{U}_{i, j}^{n+1}=\mathbf{U}_{i, j}^{n}-\frac{\Delta t}{\Omega_{i, j}} \sum_{r=1}^{4}[\mathbf{F}, \mathbf{G}]_{r} \cdot \mathbf{n}_{r} \Delta L_{r}+\Delta t \mathbf{S}_{i, j}^{n}
$$

The finite volume method, as represented by equation (17), allows the decomposition of a $2 \mathrm{D}$ problem into a series of locally one-dimensional problems to value the normal flux through every side of a cell. 
Generally the most popular finite volume schemes are upwind schemes and central schemes. In the upwind schemes the computational cells are selected according to the propagation of the perturbations while the central schemes are characterized by a central discretization of the flux vectors through a side of the cell. In particular in the analysis presented herein the HLL first order upwind scheme has been implemented and used for integrating the complete and kinematic model while MacCormack (MC) second order space centered scheme has been applied to the complete, diffusive and kinematic model. Several authors have used the MacCormack scheme to simulate the propagation of overland flow processes; among them $[2,4,5,12,13]$.

The HLL scheme only considers the left and right wave characteristics as representative of the minimum and the maximum speed of the perturbation. That scheme, applied to the $2 \mathrm{D}$ equations, gives the following expression for the numerical flux across the edge of the computational cell $\Omega_{L}$ on the left and $\Omega_{R}$ on the right:

$$
[\mathbf{f}, \mathbf{g}]_{r} \cdot \mathbf{n}_{r}= \begin{cases}{[\mathbf{f}, \mathbf{g}]_{L} \cdot \mathbf{n}_{r}} & \text { if } s_{L} \geq 0 \\ \frac{s_{R}([\mathbf{f}, \mathbf{g}])_{L} \cdot \mathbf{n}_{r}-S_{L}([\mathbf{f}, \mathbf{g}])_{R} \cdot \mathbf{n}_{r}+s_{L} s_{R}\left(\mathbf{U}_{R}-\mathbf{U}_{L}\right)}{s_{R}-s_{L}} & \text { if } s_{L} \leq 0 \leq s_{R} \\ {[\mathbf{f}, \mathbf{g}]_{R} \cdot \mathbf{n}_{r}} & \text { if } s_{R} \leq 0\end{cases}
$$

For the expressions of the wave celerities one may refer to Toro [19]. In the case of the discretization of the kinematic model, the equation (18) has been only applied to the mass conservation equation while the momentum equations, along the two directions $x$ and $y$, have been simply resolved computing the velocities through the kinematic equations using Gauckler-Strickler's formula.

MacCormack's predictor-corrector scheme has an accuracy of second order in both space and time. The numerical integration of system is performed in the following form:

$$
\begin{gathered}
\mathbf{U}_{i, j}^{p}=\mathbf{U}_{i, j}^{n}-\frac{\Delta t}{\Omega_{i, j}} \sum_{r=1}^{4}[\mathbf{F}, \mathbf{G}]_{r}^{n} \cdot \mathbf{n}_{r} \Delta L_{r}+\Delta t \mathbf{S}_{i, j}^{n} \\
\mathbf{U}_{i, j}^{c}=\mathbf{U}_{i, j}^{n}-\frac{\Delta t}{\Omega_{i, j}} \sum_{r=1}^{4}[\mathbf{F}, \mathbf{G}]_{r}^{p} \cdot \mathbf{n}_{r} \Delta L_{r}+\Delta t \mathbf{S}_{i, j}^{p} \\
\mathbf{U}_{i, j}^{n+1}=\frac{1}{2}\left(\mathbf{U}_{i, j}^{p}+\mathbf{U}_{i, j}^{c}\right)
\end{gathered}
$$

where $p$ and $c$ stand for predictor and corrector values. For each side $(r=1, \ldots, 4)$, $\mathbf{F}_{\mathrm{r}}$ and $\mathbf{G}_{\mathrm{r}}$ are obtained referring to upstream and downstream volumes alternately. The sequence is concluded in four time steps. In this paper, the TVD algorithm has been used in order to prevent the generation of numerical oscillations in the Mac Cormack scheme.

The MacCormack scheme has been applied to the diffusive model (eqs. (6)) discretizing the mass conservation equation as in the equations (19-21). For the dynamic equations the following expressions have been used: 


$$
\frac{\partial H}{\partial x}=\frac{u \sqrt{u^{2}+v^{2}}}{K_{s}^{2} h^{4 / 3}} ; \quad \frac{\partial H}{\partial y}=\frac{v \sqrt{u^{2}+v^{2}}}{K_{s}^{2} h^{4 / 3}}
$$

where $H=z+h$ is water elevation and $z$ is bed elevation. In the kinematic wave model, the momentum equations are reduced to the uniform law equations from which the values of the velocities have been computed.

\section{Applications}

The models have been validated using a number of tests commonly adopted in the literature. In particular, the numerical tests refer to situations in which the rainfall may be constant or variable both in time and space.

\subsection{Rainfall intensity constant in time and space over a plane}

This test, used for example in $[6,11]$, consists in a rainfall intensity constant in time and space $(0.33 \mathrm{~mm} / \mathrm{min})$, duration $200 \mathrm{~min}$ over a plane $400 \mathrm{~m}$ long, with constant slope (0.0005) and Manning coefficient $n=0.02 \mathrm{~s} / \mathrm{m}^{1 / 3}$.

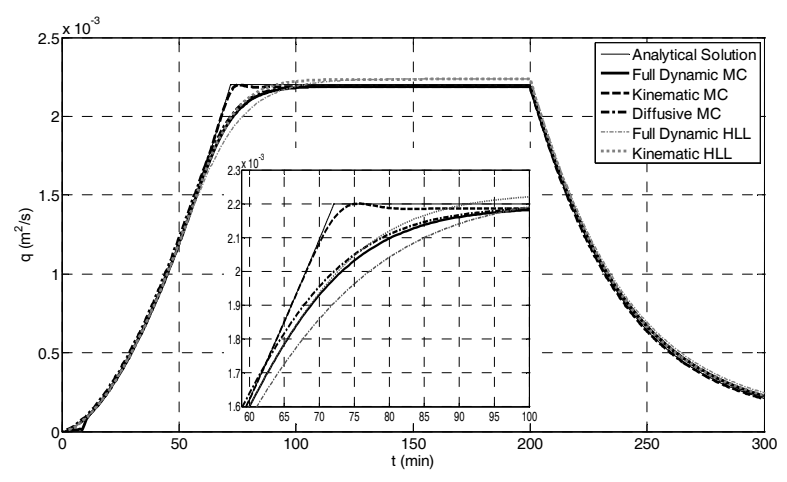

Figure 1: Comparison between the analytical and the numerical unit discharge outflow hydrographs.

For this test an analytical solution of the discharge hydrograph can be obtained using the kinematic wave theory. In figure 1, the comparison between numerical results and the analytical solution is shown. In all the simulations the computational domain has been divided in square cells of side equal to $5 \mathrm{~m}$ and the Courant number has been set equal to 0.2. As shown in figure 1 the results obtained by all schemes agree with the analytical solution. In particular MacCormack's scheme, applied to the kinematic model, gives the most accurate results while HLL scheme, applied to both the complete model and the kinematic model, presents a slight overestimation of the outflow discharge. 


\subsection{Variable in time rainfall intensity over a plane}

These tests, proposed in $[7,8]$, consist in a variable in time and constant in space rainfall intensity over a plane, $22 \mathrm{~m}$ long, with constant slope and Chézy coefficient $\chi=1.336 \mathrm{~m}^{1 / 2} / \mathrm{s}$. Two different slopes have been considered: 0.001 and 0.04. The numerical results are shown in figures 2(a) and 2(b) respectively. In these tests the numerical runoff computed by simplified models are compared with the solutions obtained by the complete models. The computational domain for both tests has been divided in the cells of dimensions $0.1 \times 0.1 \mathrm{~m}$ while the Courant number is set to 0.1 .
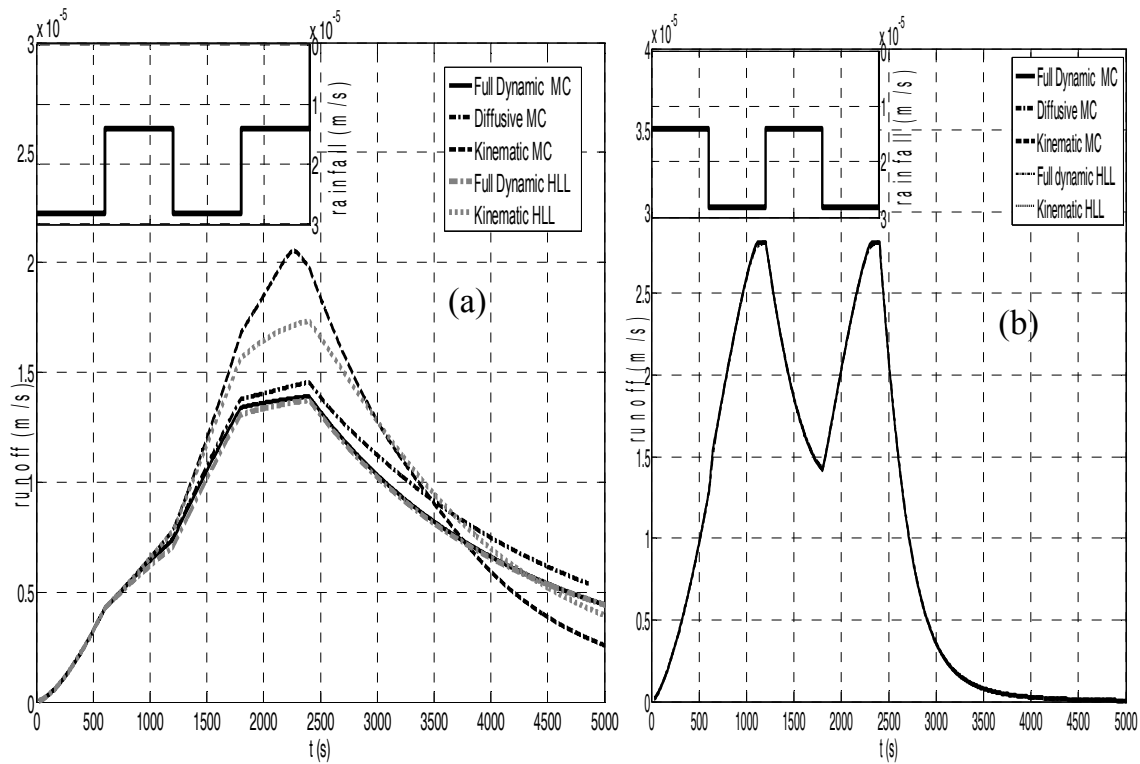

Figure 2: Comparison among the simulated runoff hydrographs at the channel outlet: slope 0.001 (a) and slope 0.04 (b).

It is interesting to observe that when using a slope equal to 0.001 the simulations are quite different and in particular the kinematic approximation gives a poor prediction because, in this case, the numerical depth gradient contribution is not negligible in comparison to the bottom slope (figure 2(a)). On the contrary, the solutions of the implemented schemes are very similar when using a slope equal to 0.04 (figure 2(b)). For both cases the numerical results agree with those presented by other authors.

\subsection{Tests concerning constant in time rainfall intensity over an ideal basin}

In this test $[1,18]$ an ideal basin, composed by two constant slope hillsides at whose bottom a constant slope channel is located, has been considered. A constant rainfall intensity $(10.8 \mathrm{~mm} / \mathrm{h})$ falls over two planes $800 \times 1000 \mathrm{~m}$, having 

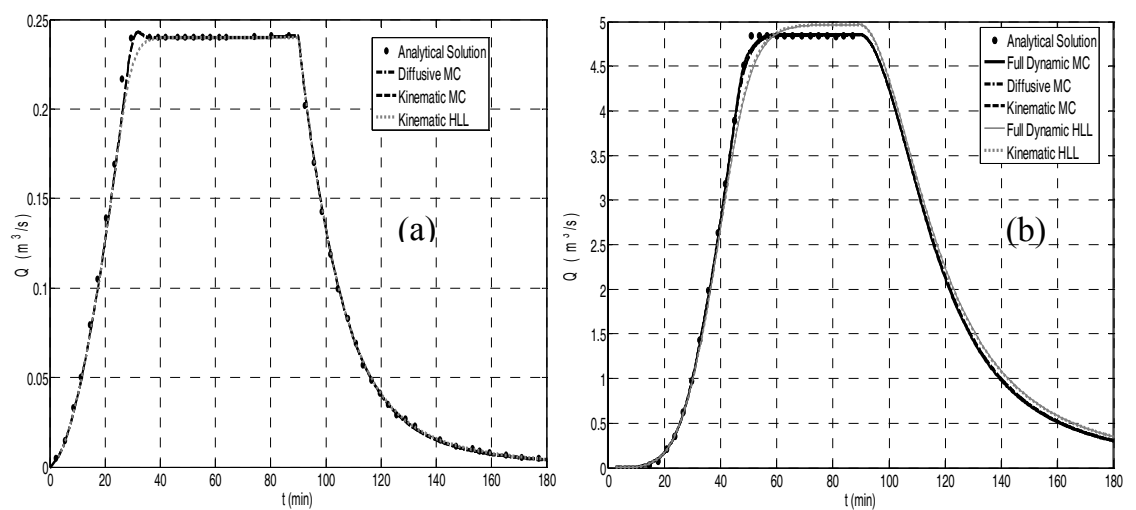

Figure 3: Comparison between numerical and analytical flood wave at the bottom of the hillside (a) and at the channel outlet (b).

Manning coefficient $\mathrm{n}=0.015 \mathrm{~s} / \mathrm{m}^{1 / 3}$, transversal slope 0.05 and no longitudinal slope, whose discharges flow into a constant slope (0.02) channel with Manning coefficient $\mathrm{n}=0.15 \mathrm{~s} / \mathrm{m}^{1 / 3}$.

Figure 3 shows the results obtained by the different schemes compared with the analytical solution in terms of both the outflow discharge coming down for each hillside and the discharge at the channel outlet. In both figures the numerical results obtained by the implemented schemes agree with the analytical solution. In particular the computed numerical solutions agree with each other except for a small diffusion caused by the first order HLL scheme with a small increase of the outflow discharge.

\subsection{Variable in space constant in time rainfall intensity over a cascade of plane}

These experiments, carried out by Iwagaki [10] and used as validation test in [3, 4], consist in a variable in space but constant in time rainfall intensity over a cascade of three planes. Each plane section was $8 \mathrm{~m}$ long, with slopes of 0.02 , 0.015 and 0.01 in the downstream directions; each section received a constant rainfall input of 389, 230 and $288 \mathrm{~cm} \mathrm{~h}^{-1}$ respectively. Discharge and water depth hydrographs are available with reference to three rainfall duration $(\mathrm{t}=10 \mathrm{~s}$, $\mathrm{t}=20 \mathrm{~s}, \mathrm{t}=30 \mathrm{~s}$ ). For every test, the computational domain has been obtained using a structured mesh with a cell size equal to $0.1 \mathrm{~m}$; the Manning coefficient was set equal to $0.01 \mathrm{~s} / \mathrm{m}^{1 / 3}$. In figures $4-5$ the comparison between the numerical results and the experimental data, relative to the shortest and to the longest rainfall duration, is shown; in particular, for each test, the water depth profiles refer to the time instant in which the rain ends $(30 \mathrm{~s}, 10 \mathrm{~s})$. Numerical results agree quite well with experimental data. In particular, as shown in figure 4(a), all the numerical hydrographs give a good prediction of the peak value. The fully dynamic wave model provides the better overall solution with reference to both the rising and recession limbs of hydrograph and to the water depth profile at the 
end of the rainfall duration (figure 4(b)); in particular, the water depth values predicted by the simplified models underestimate the experimental data. The most difficult simulation refers to the situation in which a rainfall duration equal to $10 \mathrm{~s}$ occurs because in this experiment a shock wave, which arrives at the downstream end at approximately $25 \mathrm{~s}$, is produced (Fiedler and Ramirez [4]). In figure 5, only the results relative to the MacCormack scheme are shown for the sake of clarity.
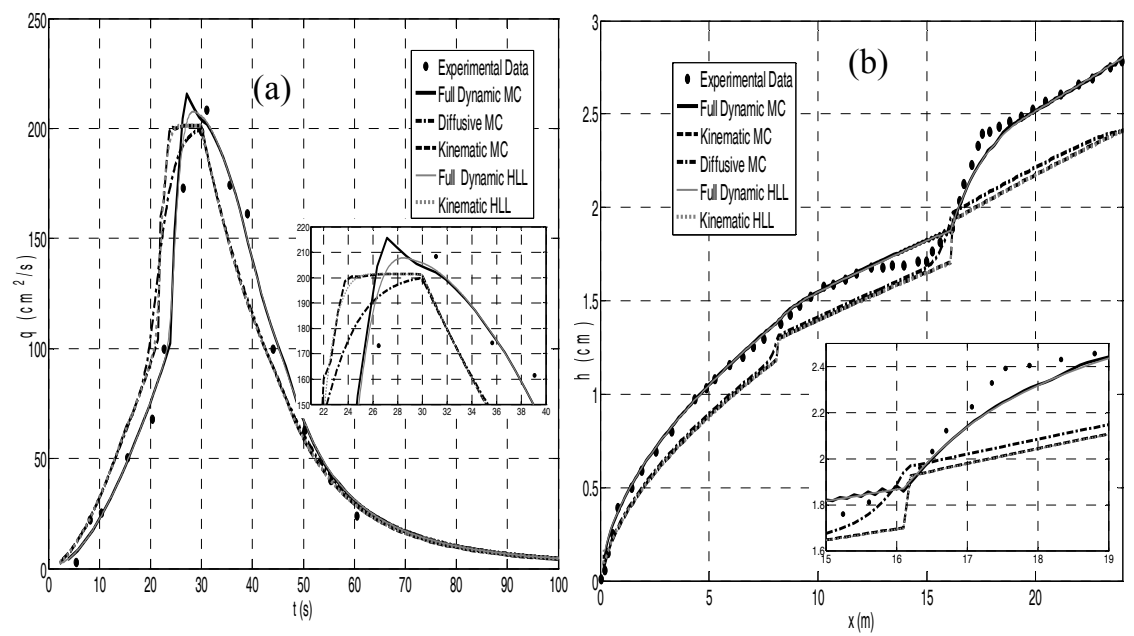

Figure 4: Comparison between experimental data and numerical results (rain duration $\mathrm{t}=30 \mathrm{~s}$ ): (a) flood wave at the channel outlet, (b) longitudinal water depths profile at the end of the rainfall input.

For this test, the numerical simulations gave different predictions of the flood wave at the end of the last plane. As to the discharge peak value, the fully dynamic and kinematic model predictions agree with the experimental one while the diffusive model provides a significant underestimation (figure 5(a)). This effect is less emphasized during the simulation reproducing the experiment shown in figure 4 because of the longer rain duration.

Once again the numerical results achieved by the implemented codes are similar to those presented in literature by other authors for the same tests. The most accurate solutions are those computed discretizing the complete models using both the HLL scheme and the MacCormack scheme.

The study of a overland flow processes in a real situation often refers to large areas; as a consequence, in order to avoid a significant increase in terms of both computational times and memory storage, the computational domain is obtained by using very coarse cells. Therefore an analysis on the accuracy of the numerical solutions in relationship to the size of computational cell has been performed; for the sake of brevity, only the fully dynamic model has been considered. In figure 6 the comparisons of the discharge hydrographs obtained 

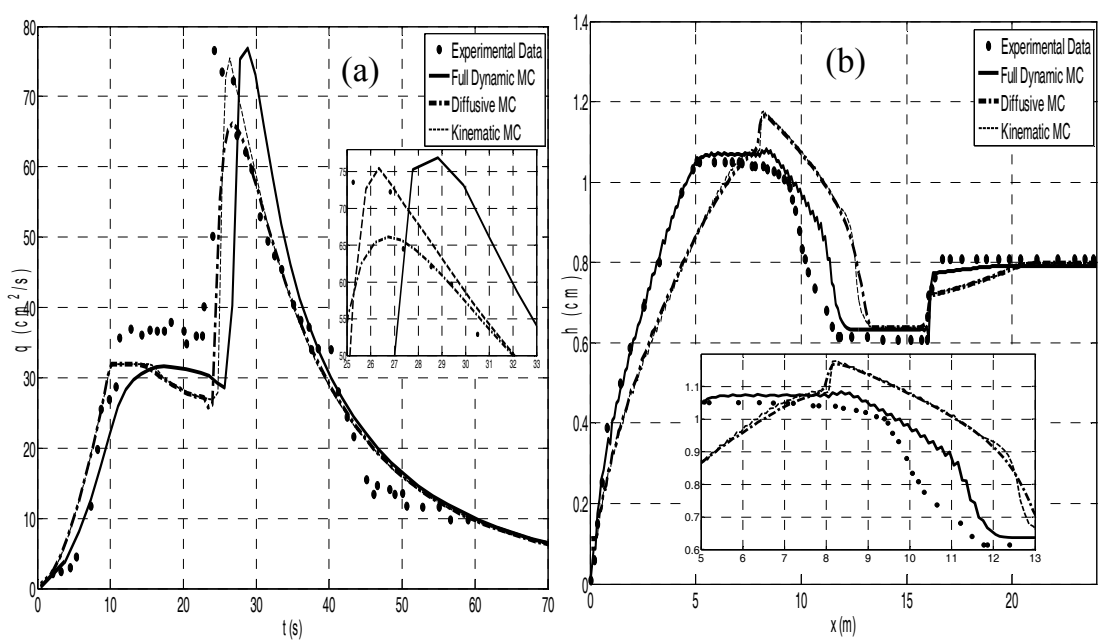

Figure 5: Comparison between experimental data and numerical results (Rain duration $\mathrm{t}=10 \mathrm{~s}$ ): (a) flood wave at the channel outlet, (b) longitudinal water depths profile at the end of the rainfall input.
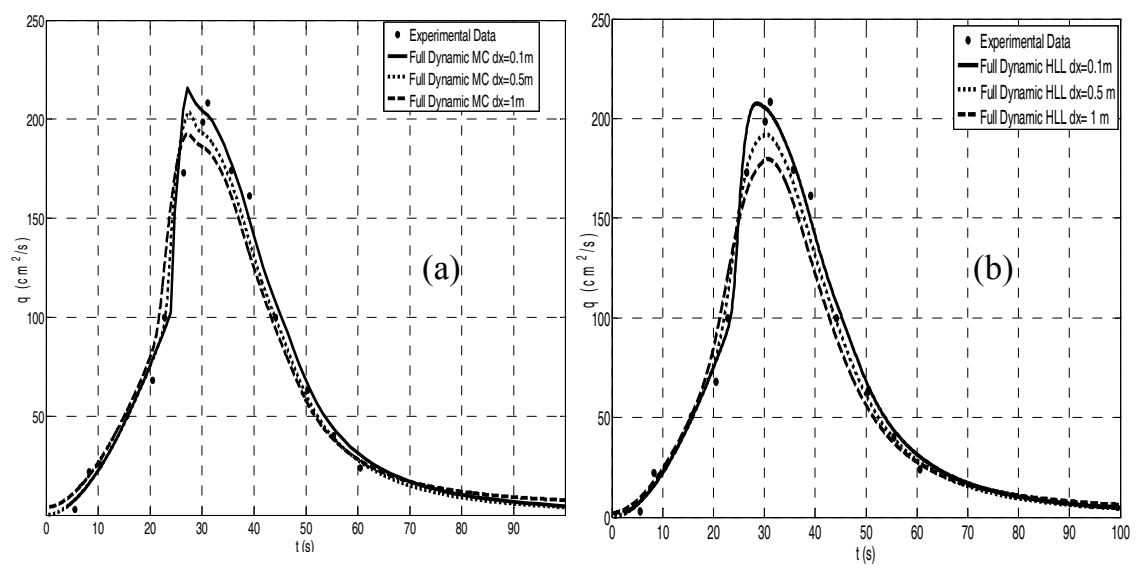

Figure 6: Flood wave at the channel outlet (Rain duration $\mathrm{t}=30 \mathrm{~s}$ ): influence of mesh size on the computed hydrographs using complete MacCormack scheme (a) and complete HLL scheme (b).

by the implemented schemes by using different cell sizes $(\Delta \mathrm{x}=0.1 \mathrm{~m}, \Delta \mathrm{x}=$ $0.5 \mathrm{~m}, \Delta \mathrm{x}=1 \mathrm{~m}$ ) are shown. It is interesting to notice that an increase of the cell size in the MacCormack scheme does not excessively change the accuracy of the solution (figure 6(a)). On the contrary, the results obtained using the HLL scheme are quite sensitive to the cell size and, in particular, they are less accurate with the cell size increase (figure 6(b)). This is mainly due to the fact that 
MacCormack's scheme is a second order accurate scheme while HLL scheme is first order accurate. Moreover, it is interesting to observe that as the phenomenon becomes more impulsive also the increase of the cell size seems to induce poorer results (figure 7).
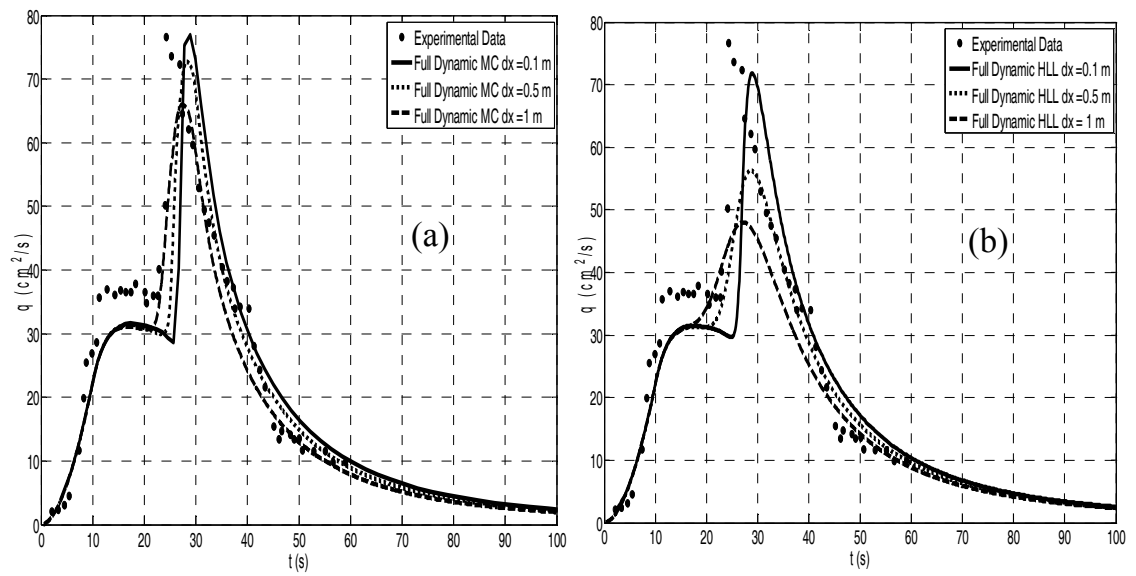

Figure 7: Flood wave at the channel outlet (Rain duration $\mathrm{t}=10 \mathrm{~s}$ ): influence of mesh size on the computed hydrographs using complete MacCormack scheme (a) and complete HLL scheme (b).

\section{Conclusions}

In this paper different overland flow models based on a $2 \mathrm{D}$ shallow water approach and relative approximation have been developed and compared. The overland flow process due to uniform rainfall over an inclined plane has been simulated in a similar way by all the models. The same behaviour has been observed when a rainfall intensity changing in time falls over a steep slope plane. When a much milder slope has been used, the kinematic wave approach has significantly overestimated, as we expected, the results obtained with the fully dynamic and the diffusive models, which still continue to give similar values. The numerical test concerning an idealised basin composed by planar element, once again did not highlight particular differences among the models. The analysis of the above-mentioned tests seems to suggest that, for overland flow simulations, the diffusive model is able to provide a very good approximation of the fully dynamic model. Indeed the results coming from the numerical simulation of the experimental test characterized by a rainfall intensity changing in space and constant in time over a cascade of three plane lead to mitigate that conclusion. In particular, the test relative to a rainfall duration of $10 \mathrm{~s}$ has shown the generation of a shock wave whose characteristics have not been adequately described by the diffusive wave model leading to a clear underestimation of the flood peak at the outlet of the last plane; this behaviour has not been observed in the kinematic model which gave results more similar to those obtained with the 
fully dynamic approach. This test suggests that the use of simplified models in situations characterized by impulsive phenomena over complex topographies may lead to important errors.

From a numeric point of view, the overall results obtained by using the MacCormack and the HLL scheme are quite good and no problems of numerical instability have been observed despite the small values of the simulated water depths. A sensibility analysis has shown that an increase of cell size causes more important negative effects on the HLL scheme than in the MacCormack scheme; this result has been expected since the MacCormack scheme has a second order of accuracy in both time and space. Moreover, as the generated flood wave becomes more impulsive also the increase of the cell size seems to cause poorer simulations.

\section{Acknowledgements}

This work was supported by CMCC - Centro Euro Mediterraneo per i Cambiamenti Climatici (Euro - Mediterranean Centre for Climate Change) in the funding project "Modellistica idraulica delle alluvioni conseguenti ad eventi meterologici intensi" (Numerical modelling of floods due to heavy rainfalls).

\section{References}

[1] Di Giammarco, P., Todini, E. \& Lamberti, P., A conservative finite elements approach to overland flow: the control volume finite element formulation. Journal of Hydrology, Elsevier, 175, pp. 276.291, 1996.

[2] Esteves, M., Faucher, X., Galle, S. \& Vauclin, M., Overland flow and infiltration modelling for small plots unsteady rain: numerical results versus observed values. Journal of Hydrology, Elsevier, 228, pp. 265-282, 2000.

[3] Feng, K. \& Molz, G. J., A 2-D diffusion-based, wetland flow model. Journal of Hydrology, Elsevier, 196, pp. 230-250, 1997.

[4] Fiedler, F. R. \& Ramirez, J. A., A numerical method for simulating discontinuous shallow flow over an infiltrating surface. International Journal of Numerical Methods in Fluids, John Wiley \& Sons Ltd, 32, pp. 219-240, 2000.

[5] Gandolfi, A. \& Savi, F., A mathematical model for the coupled simulation of surface runoff and infiltration. Journal of Agricultural Engineering Research, 75, pp. 49-55, 2000.

[6] Gottardi, G. \& Venutelli, M. A., A control-volume finite-element model for two-dimensional overland flow. Advances in Water Resources, Elsevier, 16, pp. 277-284, 1993.

[7] Gottardi, G. \& Venutelli, M., An accurate time integration method for simplified overland flow models. Advances in Water Resources, Elsevier, 31, pp. 173-180, 2008.

[8] Govindaraju, R. S., Jones, S. E. \& Kavas, M. L., On the diffusion wave model for overland flow. 1. Solution for Steep Slopes. Water Resources Research, 24 (5), pp. 734-744, 1988. 
[9] Howes, D.A., Abrahams, A. D. \& Pitman, E. B., One- and two-dimensional modelling of overland flow in semiarid shrubland, Jornada basin, New Mexico. Hydrological Processes, Wiley, 20, pp. 1027-1046, 2006.

[10] Iwagaki, Y., Fundamental studies on the runoff analysis of characteristics. Bull. 10, Disaster Prevention Research Institution, Kyoto University, Kyoto, Japan, 1955.

[11] Jaber, F. H. \& Mothar, R. H., Stability and accuracy of two dimensional kinematic wave overland flow modelling. Advances in Water Resources, Elsevier, 26, pp. 1189-1198, 2003.

[12] Kazezyilmaz-Alhan, C., \& Medina, M. A., Kinematic and diffusion waves: analytical and numerical solutions to overland and channel flow. Journal of Hydraulic Engineering, ASCE, 133(2), pp. 217-228, 2007.

[13] Liang, D., Falconer, R. A. \& Lin, B., Coupling surface and subsurface flows in a depth averaged flood wave model. Journal of Hydrology, Elsevier, 337, pp. 147-158, 2007.

[14] Moramarco, T. \& Singh, V. P., Accuracy of kinematic wave and diffusion wave for spatial-varying rainfall excess over a plane. Hydrological Processes, Wiley, 16, pp. 3419-3435, 2002.

[15] Moussa, R. \& Bacquillon, C., Criteria for the choice of flood routing methods in natural channels. Journal of Hydrology, Elsevier, 186, pp. 1-30, 1996.

[16] Ponce, V. M., Li, R. N. \& Simons, D. B., Applicability of the kinematic and diffusion models. J. Hydr. Div., Am. Soc. Civ. Eng., 104(3), pp. 353360, 1978.

[17] Sanders, B. F., Evaluation of on-line DEMs for flood inundation modelling. Advances in Water Resources, Elsevier, 30, pp. 1831-1843, 2007.

[18] Stephenson, D. \& Meadows, M.E., Kinematics Hydrology and Modelling. Elsevier, Amsterdam, 1986.

[19] Toro, E.F., Shock -Capturing Methods for Free Surface Shallow Flows. Wiley, Chichester, 2001.

[20] Tsai, W. C., Applicability of Kinematic, Noninertia, and Quasi-Steady Dynamic Wave Models to Unsteady Flow Routing. Journal of Hydraulic Engineering, ASCE, 129(8), pp. 613-627, 2003

[21] Woolhiser, D. A. \& Liggett, J. A., Unsteady, one-dimensional flow over a plane - The rising hydrograph. Water Resources Research, 3(3), pp. 753$771,1967$. 